\title{
ESTIMATION OF ISOTHERMAL DRYING RATES FOR NON-HYGROSCOPIC POROUS SLABS USING CHARACTERISTIC FUNCTIONS
}

\author{
MASAMICHI YOSHIDA, HiRonobu IMAKoma AND MoRio OKAZAKI \\ Department of Chemical Engineering, Kyoto University, Kyoto 606
}

Key Words: Drying, Characteristic Function, Nonhygroscopic Porous Material, Variable Diffusivity

A drying rate curve is influenced by the drying conditions and the nature of the material to be dried. This curve has therefore to be determined experimentally or estimated theoretically.

In a previous paper ${ }^{1)}$, an ideal drying process with a constant and uniform body temperature was investigated from slabs of a non-hygroscopic porous material. The failling-rate period can be divided into two periods, which are found theoretically to show separate relationships between the drying rate and the mean moisture content. Such relationships are called "characteristic functions".

On the basis of the characteristic functions, this paper proposes a new method for estimating drying rate curves for the above ideal process. The method allows one to estimate curves under various drying conditions from a single experimental curve, even when there is no information about the moisture diffusivity.

\section{Basic Theory}

Consider a single-sided drying process of non-hydroscopic porous slabs. The body temperature is constant and uniform. The process is assumed to be governed by the following drying mechanism: There exists a transitional moisture content $m_{b}$ below which water connot flow in liquid state and the moisture diffusivity becomes zero. The constant-rate period

\footnotetext{
* Received November 7, 1989 Correspondence concerning this article should be addressed to $M$. Yoshida.
}

ceases when the moisture content of the exposed surface reaches $m_{b}$. This period is followed by the falling-rate period, during which the material can be divided into dry and wet zones. At their boundary, called the evaporating plane, the water changes phase. The drying rate of the falling-rate period is determined by the location of the evaporating plane:

$$
\frac{F}{F_{0}}=\frac{n}{n_{0}}=\frac{1}{1+B i(1-\eta)}
$$

where the drying intensity $F_{0}$ and the flux parameter $F$ are reduced drying rates of the constant- and falling-rate periods respectively, $B i$ the Biot number of mass transfer and $\eta$ the reduced thickness of the wet zone.

The falling-rate period of the above drying process can be divided into two periods, differing in the relationship between the drying rate and the mean moisture content. Such relations are called "characteristic functions". Their features are summarized below.

The modified flux parameter and the modified mean moisture content are defined respectively as:

$$
\begin{aligned}
& \hat{F} \equiv F\left(\eta+\frac{\bar{m}+m_{b} \eta}{2} \frac{d \eta}{d \bar{m}}\right) \\
& \hat{m} \equiv \frac{\bar{m}}{\eta}
\end{aligned}
$$

Each process corresponds to a complete curve of $\hat{F}$ vs. $\hat{m}$. Curves of $\hat{F}$ vs. $\hat{m}$ of the same material under 
different drying conditions converge in the lower range of $\hat{m}$. This implies that a period exists during which the relation between $\hat{F}$ and $\hat{m}$ is independent of the drying conditions and determined only by the moisture diffusivity:

$$
\hat{F}=\text { func. }\left(\hat{m} ; D_{r}\right)
$$

This period is defined as the regular regime. The relation between $\hat{F}$ and $\hat{m}$ for this period, represented by that convergence curve, is called the regular regime function.

In general, the whole falling-rate period belongs approximately to the regular regime. When $F_{0}$ has an extremely high value, however, the regular regime is preceded by a period during which the following relation is valid instead of Eq. (4):

$$
F\left(m_{0}-\bar{m}+\frac{m_{0}}{B i}\right)=\gamma=\text { const . }
$$

where $\gamma$ depends on both the drying condition and the moisture diffusivity. This is called the penetration period. Differentiating Eq. (5) with respect to $\bar{m}$ leads to an equation which does not include the unknown constant $\gamma$ :

$$
G=\frac{\bar{m}}{m_{0}-\bar{m}+\left(m_{0} / B i\right)}
$$

where $G$ is a variable defined as:

$$
G \equiv \frac{d(\ln F)}{d(\ln \bar{m})}
$$

This relation between $G$ and $\bar{m}$, valid for this period, is called the penetration period function. The unknown constant $\gamma$ can be determined by this function, as discussed later.

\section{Procedure for Estimation of the Drying Rate Curve}

In this section, based on the two characteristic functions, a new method is proposed for estimation of drying rate curves of the process described above. Note that drying rate curves under various drying conditions can be obtained from a single experimental curve with no information about the moisture diffusivity. The values of $\bar{D}_{e v}$ and $m_{b}$, necessary for estimation, can be determined from the experimental curve as well. The estimation procedure consists of the following steps.

Step 1: Measurement of a drying rate curve Obtain a drying rate curve by experiment. One can use it as the reference to determine transport properties and the regular regime function. The experiment should be done with a drying intensity higher than or equal to the maximum value of interest. The larger the ratio of $F_{0} / B i$, the more accurately can $m_{b}$ be determined.
Step 2: Determination of $B i$ When the mean moisture content reaches zero, the wet zone vanishes. Substituting $\eta=0$ into Eq. (1) hence yields an expression for $F_{f n}$, the limiting value of the drying rate as $\bar{m} \rightarrow 0$ :

$$
F_{f n}=\frac{F_{0}}{1+B i}
$$

Since $F_{f n}$ is obtained by extrapolating the drying rate curve, $B i$ can be determined from Eq. (7). $\bar{D}_{e v}$ is then determined from the definition of the Biot number.

Step 3: Drawing a curve of $\eta$ vs. $\bar{m}$ Since $B i$ is now a known parameter, $\eta$ can be calculated from Eq. (1) for a given $F$. Thus one can draw a curve of $\eta$ vs. $\bar{m}$ for the experiment. This curve is indispensable in determining $m_{b}$ and the regular regime function.

Step 4: Determination of $m_{b}$ When the ratio of $F_{0} / B i$ is large enough, the moisture content profile becomes almost flat at the end of the process. This implies that the curve of $\eta$ vs. $\bar{m}$ finally coincides with a line expressed as $\eta=\bar{m} / m_{b}{ }^{1}$. The value of $m_{b}$ can hence be determined by drawing a tangent line passing through the origin on the curve of $\eta$ vs. $\bar{m}$ obtained in the previous step.

Step 5: Drawing the regular regime function Values of $\hat{F}$ and $\hat{m}$ can be calculated according to their definition by using the drying rate curve and the curve of $\eta$ vs. $\bar{m}$. Thus one can obtain a curve of $\hat{F}$ vs. $\hat{m}$, which is the regular regime function except a part corresponding to the penetration period during which Eq. (5) is valid.

Step 6: Setting drying condition Express a target drying condition in terms of $F_{0}, B i$ and $m_{b}$.

Step 7: Drawing drying rate curve of regular regime Equation (2) can be rewritten as:

$$
\frac{d \eta}{d \bar{m}}=-2 \frac{\hat{F}-F \eta}{F\left(\bar{m}+m_{b} \eta\right)}
$$

This is an ordinary differential equation of $\eta$ depending on $\bar{m}$. Since the regular regime function is already known and $F$ is given by Eq. (1) as a function of $\eta$, one can solve Eq. (8) to obtain the drying rate curve of the regular regime for the target drying condition. The initial condition is

$$
\eta=1 \quad \text { at } \quad \bar{m}=\bar{m}_{c r}
$$

where the critical moisture content $\bar{m}_{c r}$ is an unknown parameter in this study and hence must be estimated. This can be done by solving Eq. (8) with an assumed $\bar{m}_{c r}$, because the behavior of the solution tells one whether the assumed $\bar{m}_{c r}$ is proper or not; when Eq. (8) is solved with a $\bar{m}_{c r}$ value higher than the true value, the resultant drying rate curve shows a maximum. On the other hand, which a lower $\bar{m}_{c r}$ value the curve shows a discontinuity. With the help of these criteria 
one can determine $\bar{m}_{c r}$ by trial and error.

Step 8: Drawing drying rate curve of penetration period The relation between $G$ and $\bar{m}$ of the penetration period is given by Eq. (6) irrespective of the material to be dried. On the other hand, that of the regular regime can be obtained from the drying rate curve of this period drawn in the previous step. It is assumed here that the transition from the penetration period to the regular regime occurs at the intersection of these two curves. The constant $\gamma$ in Eq. (5) is then determined by substituting values of $G$ and $\bar{m}$ at the intersection into Eq. (5). Then the drying rate curve of the penetration period is readily drawn by using this equation. When $F$ at the intersection is greater than $F_{0}$, the penetration period does not exist for the target condition.

Step 9: Completion of drying rate curve The whole drying rate curve for the target drying condition can be obtained by connecting three curves, i.e., drying rate curves of the regular regime and the penetration period obtained in the previous steps, and that of the constant rate period, $F=F_{0}$.

\section{Results and Discussion}

The validity of the present method is examined through direct numerical solutions of the mass transfer equations given in the previous paper. ${ }^{1)}$ From a numerical drying rate curve chosen as the reference, curves with different drying conditions are estimated by applying this method. Note that no information about the moisture diffusivity is used in the estimation. Figures 1 and 2 show typical comparisons of estimated curves and numerical ones to prove the present method to be valid.

Established under an ideal isothermal condition, the present method cannot directly apply to practical non-isothermal cases. Yet, since the method has an attractive advantage of allowing one to estimate drying rate curves from a single experimental curve with no information about the moisture diffusivity, the method may become a powerful tool when extended to practical processes. This extension may be difficult but is not impossible.

\section{Nomenclature}

\begin{tabular}{|c|c|}
\hline$B i$ & $\begin{aligned}= & \text { Biot number of mass transfer } \\
& \left(=k_{g} L /\left(M_{v} \bar{D}_{e v} / R T\right)\right)\end{aligned}$ \\
\hline $\bar{D}_{e v}$ & $=$ effective diffusivity of vapor \\
\hline$D_{l}$ & $=$ moisture diffusivity \\
\hline$D_{l 0}$ & $=$ arbitrary reference value of $D_{l}$ \\
\hline$D_{r}$ & $\begin{aligned}= & \text { dimensionless moisture diffusivity } \\
& \left(=D_{l} / D_{t 0}\right)\end{aligned}$ \\
\hline 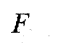 & $=$ flux parameter $\left(=n L /\left(D_{l 0} \rho_{m}^{*}\right)\right)$ \\
\hline$F_{0}$ & $=$ drying intensity $\left(=n_{0} L /\left(D_{10} \rho_{m}^{*}\right)\right)$ \\
\hline$\hat{F}$ & $\begin{aligned}= & \text { modified flux parameter } \\
& \left(=F\left\{\eta+\left(\bar{m}+m_{b} \eta\right)(d \eta / d \bar{m}) / 2\right\}\right)\end{aligned}$ \\
\hline & $=$ parameter $(=d(\ln F) / d(\ln \bar{m}))$ \\
\hline & $=$ film mass transfer coefficient \\
\hline
\end{tabular}

$k_{g} \quad=$ film mass transfer coefficient

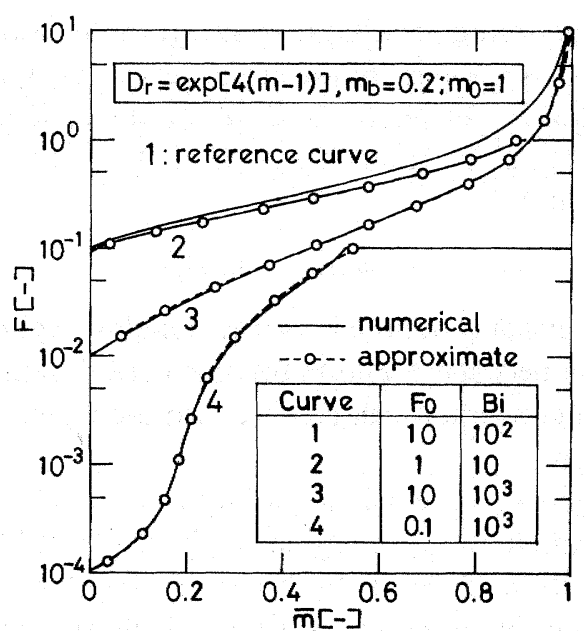

Fig. 1. Drying rate curves; $D_{r}=\exp \{4(m-1)\}, m_{b}=0.2$ and $m_{0}=1$.

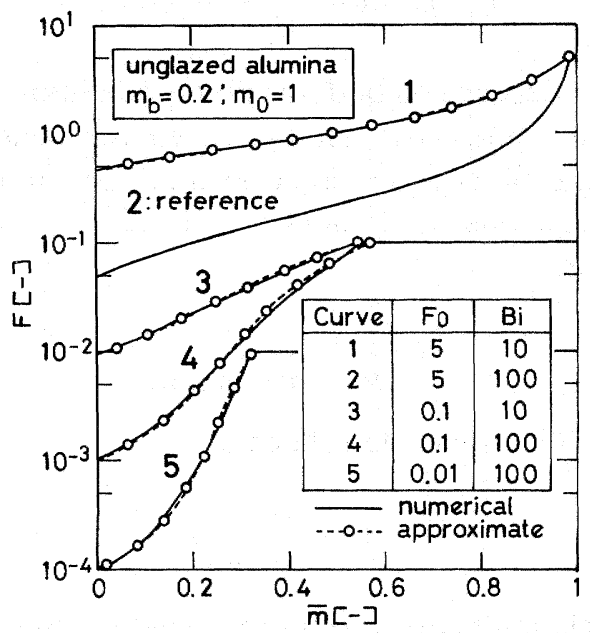

Fig. 2. Drying rate curves; $D_{r}=\exp \left\{\sum_{n=0}^{6} a_{n} m^{n}\right\}, m_{b}=0.2$ and $m_{0}=1 . a_{n}(n=0,1, \cdots, 6):-20.07,112.0,-252.8,244.6$, $-83.73,4.937 \times 10^{-4},-6.751 \times 10^{-4}$

$L \quad=$ thickness of material $[\mathrm{m}]$

$M_{v} \quad=$ molecular weight of vapor $[\mathrm{kg} / \mathrm{mol}]$

$=$ mean moisture content $\left(=\bar{\rho}_{m} / \rho_{m}^{*}\right)$

$=$ modified mean moisture content $(=\bar{m} / \eta)$

$=$ transitiona- $l$ moisture content

$=$ critical moisture content

$=$ initial moisture content

$=$ drying rate

$=$ constant drying rate

$=$ gas constant

$=$ abosolute temperature

$=$ constant in Eq. (5)

$=$ ratio of the thickness of the wet zone to that of the material

$=$ mass concentration of moisture 


$$
=\text { saturated }
$$

\section{Literature Cited}

1) Yoshida, M., H. Imakoma and M. Okazaki: J. Chem. Eng.

\section{Japan, 23, 58 (1990).}

(Presented at the 6th International Drying Symposium, at Versailles, France, September 1988. 